\title{
Une nouvelle mégaclassification pragmatique du vivant
}

Damien Aubert

> Classer les espèces et reconstruire l'histoire évolutive sont des activités intimement reliées, mais la nature exacte de leur relation fait toujours débat. Leurs disciplines respectives - la taxonomie et la phylogénétique - poursuivent parfois des objectifs divergents, ce qui peut être source de tensions comme l'illustre une révision récente de la classification du vivant. <

Depuis 2001, le projet international Species $2000^{1}$ collabore avec l'organisation nord-américaine Integrated taxonomic information system (ITIS) afin de recenser toutes les espèces connues dans leur Catalogue of life (CoL). Un peu plus de 1,6 million d'espèces ont déjà été listées à ce jour. Cette énorme masse de données est utile à la recherche sur la biodiversité ainsi qu'aux initiatives de sauvegarde de celle-ci, mais elle peut également permettre de faire progresser les sciences médicales (la parasitologie par exemple) ou même l'agriculture [1]. Elle nécessite donc un système de classement consensuel afin de stocker et de retrouver facilement les informations pertinentes. La publication en avril 2015 d'une nouvelle classification à grande échelle (ou mégaclassification) [2] marque donc une étape importante de ce projet. Les auteurs n'ont pas opté pour une «classification phylogénétique », c'està-dire un classement suivant la doctrine cladiste ${ }^{2}$ qui stipule que tous les groupes doivent inclure l'ensemble des descendants de leur ancêtre commun [3]. De tels groupes sont alors dits holophylétiques ${ }^{3}$ (Figure 1).

Vignette (Photo @ Inserm/Musée Jules Verne-Ville de Nantes/Éric Dehausse). ${ }^{1}$ http://www.sp2000.org/

${ }^{2}$ École de taxonomie reposant sur la construction de groupes (alors appelés clades, du grec clados, qui signifie «branche ») incluant un ancêtre commun et l'ensemble de ses descendants.

${ }^{3}$ Un groupe est dit holophylétique s'il comprend seulement une espèce mère et toutes les espèces qui en descendent.

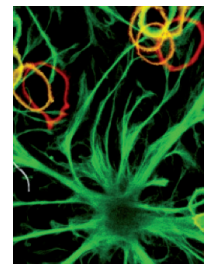

Académie de Clermont-Ferrand, 3, avenue Vercingétorix, 63033 Clermont-Ferrand, France. damien.aubert@ac-clermont.fr

Largement dominant, le cladisme n'est cependant pas consensuel parmi les taxonomistes. En effet, le rythme de l'évolution étant très hétérogène d'une branche à l'autre, les groupes holophylétiques sont parfois morphologiquement très disparates. Au contraire, certaines espèces ayant des phénotypes plus proches ont parfois un ancêtre plus ancien (Figure 1). Certains taxonomistes préfèrent donc l'école évolutionniste qui autorise les groupes paraphylétiques, c'est-à-dire excluant les descendants ayant trop divergé [4]. En pratique une classification évolutionniste est moins soumise à

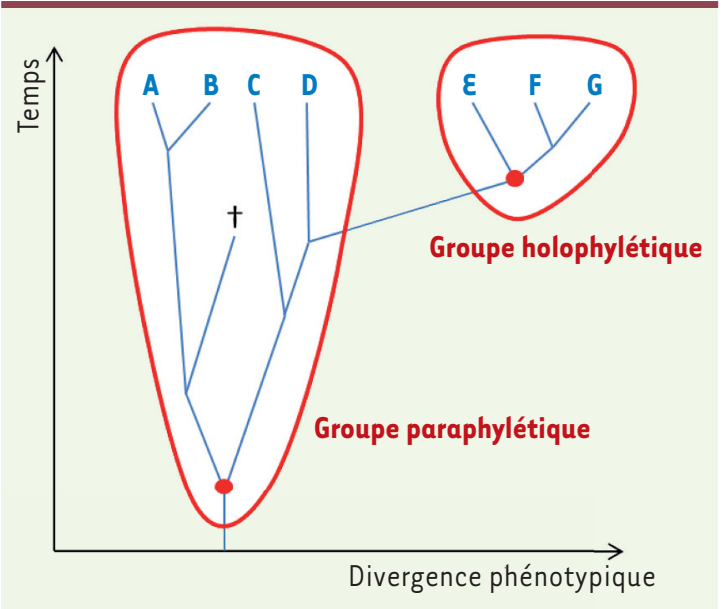

Figure 1. Définitions des relations phylogénétiques. L'obèle représente une lignée éteinte, les lettres des espèces actuelles, les points rouges le dernier ancêtre commun des membres de chaque groupe. On note qu'un groupe holophylétique contient tous les descendants de son ancêtre commun contrairement à un groupe paraphylétique. 

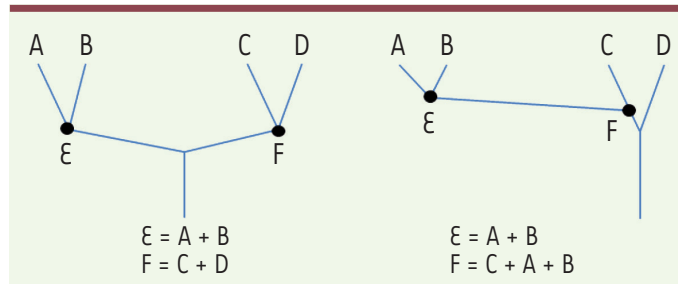

$\varepsilon=A+B$

$F=C+A+B$
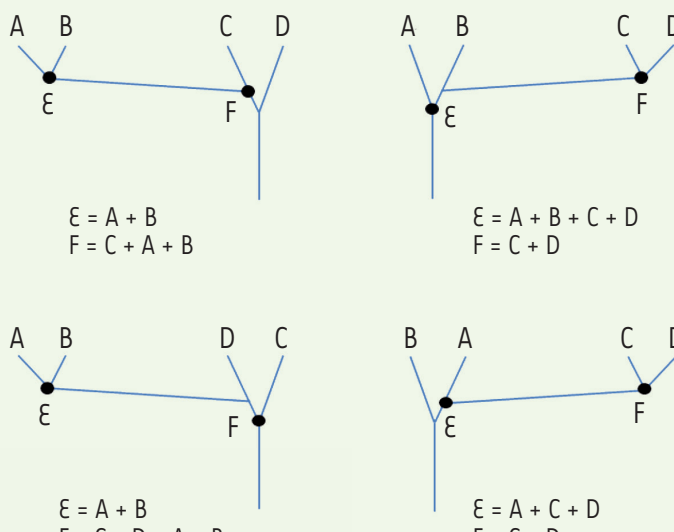

$F=C+D+A+B$

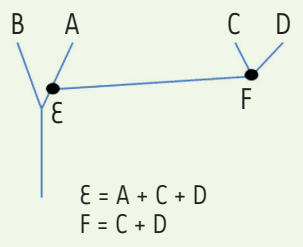

Figure 2. Instabilité de la classification phylogénétique. Deux familles de deux espèces, $\varepsilon=A+B$ et $F=C+D$ (l'espèce $A$ est le type de la famille $\varepsilon$, tandis que $C$ est celui de $F$ ), sont morphologiquement très distinctes, mais leurs relations phylogénétiques sont mal connues. Alors qu'une classification autorisant les groupes paraphylétiques restera stable dans toutes les configurations, une classification rejetant la paraphylie verra les définitions des groupes $\varepsilon$ et $F$ changer en fonction des hypothèses concernant l'ordre des ramifications.

l'instabilité due aux hypothèses fluctuantes concernant la structure exacte de l'arbre du vivant [5]. En effet, quel que soit l'ordre exact des ramifications menant aux différentes espèces, les groupes fondés à la fois sur une ascendance commune et une similarité morphologique resteront stables, alors que les groupes définis seulement comme une communauté de descendants verront leurs définitions modifiées (Figure 2). On peut de plus argüer que la paraphylie est inévitable. Quelle que soit la manière dont on s'y prenne, couper une branche (holophylétique) de l'arbre de la vie génère automatiquement une souche (paraphylétique) [6]. Le problème est plus évident pour les paléontologues car ces derniers sont parfois capables de reconnaître l'espèce mère d'une ou plusieurs espèces filles [7].

\section{La proposition de classification}

Après avoir consulté plus de 3000 experts d'horizons divers, aussi bien cladistes qu'évolutionnistes, l'équipe du Catalogue of life (CoL) a donc préféré une classification à la fois plus stable et plus pratique. Bien que cette classification autorise la paraphylie, les auteurs ne se réclament pas explicitement de l'école évolutionniste. On peut cependant remarquer que leur système est une extension et une mise à jour de la mégaclassification datant de 1998 proposée par Thomas Cavalier-Smith (un auteur indiscutablement évolutionniste) [8]. Ainsi, plutôt que l'hypothèse controversée des trois domaines Archaea, Bacteria et Eucarya ${ }^{4}$ [9], on retrouve dans la classification du CoL les deux empires traditionnels que sont les procaryotes (Prokaryota) et les eucaryotes (Eukaryota). Le premier comprend deux règnes, les bactéries (Bacteria) et les archées (Archaea), tandis que le second

${ }^{4}$ Théorie systématique selon laquelle le monde vivant serait divisible en trois lignées très distinctes ayant divergé très tôt dans l'histoire de la vie. se subdivise en cinq règnes, les protozoaires (Protozoa), les animaux (Animalia), les champignons (Fungi), les plantes (Plantae) et les chromistes (Chromista). Thomas Cavalier-Smith étant I'un des co-auteurs de cette classification, il est assez tentant de faire correspondre ces sept règnes aux phylogénies qu'il propose [10] (Figure 3). Les noms de ces règnes sont pour la plupart connus, mais les chromistes restent souvent méconnus du grand public et même des biologistes non spécialisés. Ils regroupent pourtant de nombreuses espèces célèbres comme le goémon ou varech (un mélange d'algues brunes très répandues sur les côtes de Bretagne et de Normandie entre autres), les diatomées, les paramécies et même des agents pathogènes comme les oomycètes responsables du mildiou (une affection touchant la vigne, la pomme de terre, les tomates, etc.) ou encore comme les apicomplexés responsables de maladies humaines comme la toxoplasmose (Toxoplasma gondii) ou le paludisme (genre Plasmodium). Ces sept règnes sont divisés en pas moins de 96 embranchements ! Un tel nombre pourrait être révélateur d'une inflation taxonomique. On remarque en effet, en examinant la classification dans le détail, que parmi les 29 embranchements bactériens, 18 ne comportent qu'une seule classe. Cela est bien sûr en partie dû à un manque de résolution des études phylogénétiques moléculaires, mais on pourrait aussi soupçonner un effet pervers de la course aux publications qui pousse les bactériologistes à exagérer l'importance des groupes qu'ils étudient (il est bien plus vendeur de prétendre étudier un embranchement plutôt qu'une classe ou même un ordre). En témoignent par exemple les planctomycètes, les verrucomicrobes et les chlamydiées, tous les trois élevés au rang d'embranchement alors qu'il est dorénavant admis qu'ils ont une affinité commune (ils forment l'embranchement Planctobacteria dans le système de Thomas Cavalier-Smith).

\section{Perspectives taxonomiques}

La taxonomie souffre d'une mauvaise image. Elle est souvent regardée comme une discipline poussiéreuse, les taxonomistes ne sont guère nombreux et leurs départements n'ont que peu de moyens. L'étude de nombreux groupes d'espèces, parfois des embranchements entiers, repose sur l'expertise de seulement quelques chercheurs dans le monde entier. Pourtant la 


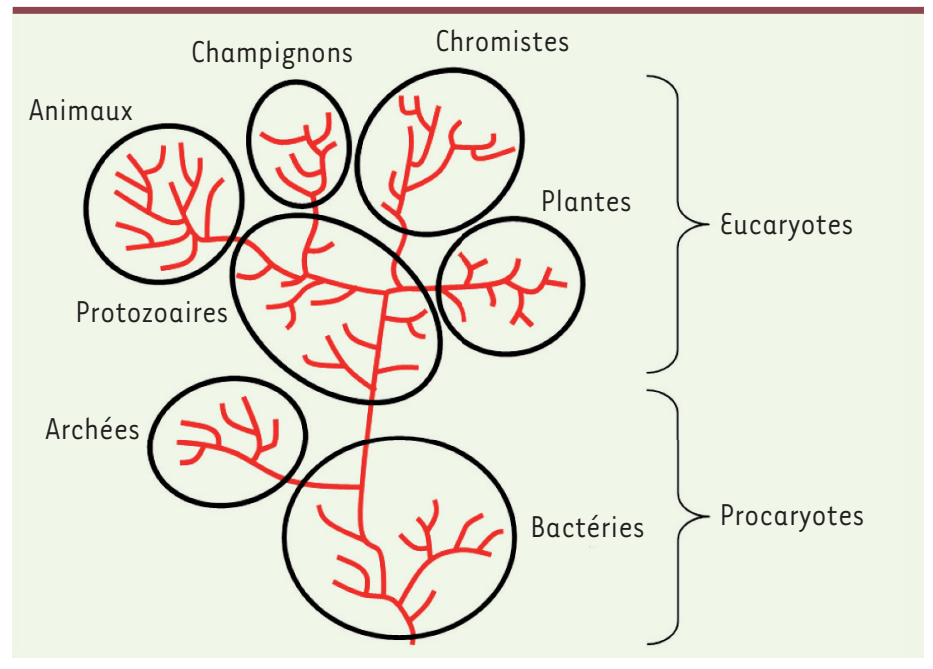

Figure 3. L'arbre de la vie. Dessiné d'après les phylogénies proposées par Cavalier-Smith [10] et la mégaclassification de Ruggiero et al. [2].

taxonomie est une discipline se situant clairement en amont de toute étude significative dans les autres domaines de la biologie. Le nom scientifique d'un organisme est une étiquette fonctionnelle permettant d'accéder à n'importe quelle information concernant celui-ci. Les programmes de santé publique ou de conservation de la faune et de la flore resteraient vains si les espèces étudiées restaient non identifiées, mal identifiées ou incorrectement classées dans les bases de données [1]. Cela doit nous faire réfléchir à notre manière de percevoir et d'enseigner la classification du vivant. Est-il plus important qu'elle reflète fidèlement une théorie scientifique particulière $[3,11]$ $(\rightarrow)$ ou bien qu'elle serve avant tout d'outil aux autres disciplines, donnant ainsi la priorité à un système de référence à la fois stable et flexible $[2,5]$ ? Le degré avec lequel la classification des taxonomistes doit refléter les arbres des phylo-

\section{$(\rightarrow)$ Voir le Forum} de D. Casane et

P. Laurenti, $m / s n^{\circ} 12$, décembre 2012, page 1121 généticiens est peut-être un mélange complexe de philosophie et de pragmatisme [4]. Mais puisqu'une seule classification générale ne

semble pas pouvoir satisfaire les exigences de tout le monde, on serait tenté de proposer que deux classifications spécialisées fonctionnant en parallèle feraient un bien meilleur travail [6]. $\diamond$

\section{A new pragmatic megaclassification of life}

\section{LIENS D'INTÉRÊT}

L'auteur déclare n'avoir aucun lien d'intérêt concernant les données publiées dans cet article.

\section{RÉFÉRENCES}

1. Tahseen $Q$. Taxonomy. The crucial yet misunderstood and disregarded tool for studying biodiversity. J Biodivers Endanger Species $2014 ; 2: 128$

2. Ruggiero MA, Gordon DP, Orrell TM, et al. A higher level classification of all living organisms. PLoS One 2015; 10 : e0119248.

3. Lecointre G, Bonnet ML, Cariou F, et al. Comprendre et enseigner la classification du vivant, $2^{\mathrm{e}}$ ed revue et augmentée. Paris : Belin, 2008 $352 \mathrm{p}$.

4. Stuessy TF. Schools of data analysis in systematics are converging, but differences remain with formal classification. Taxon $2013 ; 62: 876-85$.

5. Mayr $\varepsilon$, Bock WJ. Classifications and other ordering systems. J Zool Syst Evol Res $2002 ; 40: 169-94$

6. Aubert D. A formal analysis of phylogenetic terminology: towards a reconsideration of the current paradigm in systematics. Phytoneuron $2015 ; 66: 1-54$.

7. Paul CRC. The recognition of ancestors. Hist Biol $1992 ; 6: 239-50$.

8. Cavalier-Smith T. A revised six-kingdom system of life. Biol Rev Camb Philos Soc $1998 ; 73: 203-66$

9. Woese CR, Kandler 0 , Wheelis ML. Towards a natural system of organisms: proposal for the domains Archaea, Bacteria, and Eucarya. Proc Natl Acad Sci USA $1990 ; 87: 4576-9$

10. Cavalier-Smith T. Early evolution of eukaryote feeding modes, cell structural diversity, and classification of the protozoan phyla Loukozoa, Sulcozoa, and Choanozoa. Eur J Protistol 2013 ; 49 : 115-78.

11. Casane D, Laurenti P. Penser la biologie dans un cadre phylogénétique : l'exemple de l'évolution des vertébrés. Med Sci (Paris) 2012 ; 28 : 1121-7.

\section{TIRÉS À PART}

D. Aubert

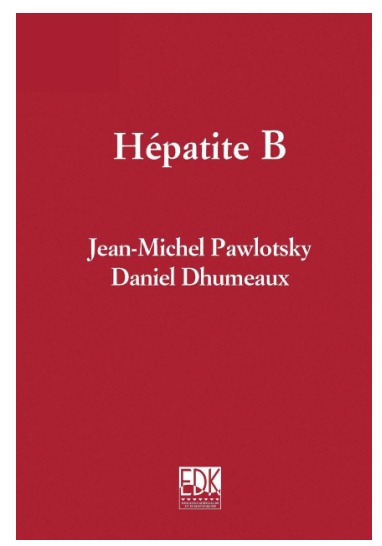

ISBN : 978-2-8425-4131-6 576 pages

\section{Bon de commande}

À retourner à EDK, 109, avenue Aristide Briand, 92541 Montrouge Cedex Tél. : 0141177405 - Fax : 0149850345 - E-mail : edk@edk.fr

NOM :

Prénom .

Adresse :

Code postal :

Ville :

Pays :

Fonction :

Je souhaite recevoir l'ouvrage Hépatite B : $54 €+3 €$ de port $=\mathbf{5 7} €$ TTC offre exceptionnelle réservée aux abonnés à $\mathrm{m} / \mathrm{s}$ jusqu'au 31 décembre 2010

en ................. exemplaire, soit un total de ....................................... €
$\square$ Par chèque, à l'ordre de $\mathbf{E} \mathbf{D}$ K
$\square$ Par carte bancaire :

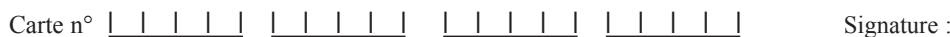

Date d'expiration: $\quad \underline{1 \mid} \underline{1 \mid 1}$

$\mathrm{N}^{\circ}$ de contrôle au dos de la carte : 